\title{
Estudio de ritual Lalay Cañari celebrado en los Andes de sur de Ecuador
}

\author{
Study of Lalay Cañari ritual celebrated in the southern Andes of Ecuador \\ Juan Carlos Simbaina Solano ${ }^{1}$ \\ Jacinto Aguaiza Quizhpilema²
}

Fecha de recepción: 24-09-2019

Fecha de aceptación: 3-02-2020

\begin{abstract}
Resumen
En la cultura Cañari de Ecuador, desde hace siglos, se ha venido realizando el ritual Lalay Cañari, el cual se ha transmitido ancestralmente con sabiduría. Sin embargo, debido a la conquista inca y española, el ritual ha perdido su esencia. Los historiadores por otra parte lo describen relacionando este con mitos y leyendas. En el presente estudio, se planteó revelar los "misterios" sobre el ritual. Para ello, se realizó una recopilación de pruebas e información mediante entrevistas y encuestas a los habitantes nativos de Cañar, a partir de los datos obtenidos, se llevó a cabo el análisis estadístico.

Palabras clave: Celebración, Ritual, Cañari, Carnaval, Lalay.
\end{abstract}

\begin{abstract}
In the Cañari culture of Ecuador, the Cañari Lalay ritual has been performed for centuries, transmitted with wisdom for generations by the ancestors. However, due to Inca and Spanish conquest, the ritual has lost its essence. Historians describe relating to myths and legends. In the present paper, a study was proposed to reveal mysteries about the ritual. To do this, a compilation of tests and information was carried out through interviews and surveys to the native inhabitants of Cañar. With the obtained data, the statistical analysis was performed.
\end{abstract}

Keywords: Celebration. Ritual. Cañari. Carnival. Lalay.

1 Investigador asociado de la Universidad del Azuay, Cuenca, Ecuador. Principales líneas de investigación Genética y Genómica animal, y pesquisa en Costumbres y Tradiciones de la Cultura Cañari. Correo electrónico: austrogenetica@gmail.com

2 Investigador asociado de la Universidad del Azuay, Cuenca, Ecuador. Principales líneas de investigación en Plantas medicinales; Cultura Cañari, Legua Kichwa y Cañari como didáctica comunitaria. Correo electrónico: jaguaizaq@gmail.com 


\section{Introducción}

El pueblo Cañari del Ecuador, es una cultura antigua que ha forjado su propia nación desde épocas milenarias (Lynch y Pollock, 1981), y su asentamiento fue en las actuales provincias de Cañar y Azuay (Iglesias, 1985; Gómez, 2003). No obstante, en los periodos de conquista inca y española, se ha perdido su idioma originario (Burgos, 2003). Sin embargo, el pueblo Cañari, ha conservado sus costumbres y tradiciones autóctonas, y ha venido manteniéndose hasta nuestros días (Alulema y López, 2017). Una de las principales costumbres es el Lalay Cañari o también conocido como carnaval Cañari (Zhungri, 2017). El denominado Lalay Cañari, es un ritual que se realiza año tras año en los días del carnaval (Morocho y Zaruma, 2012; Alulema y Guamán, 2012), y es finalizado antes del miércoles de ceniza (McGuffin, 2014). El Lalay Cañari, en la actualidad, también ha tomado adopción con el nombre de Pawkar Raymi, que quiere decir "fiesta colorida" (Matínez, 2015). Adicionalmente, en la zona norte del Ecuador el Pawkar raymi también es conocido Sisay pacha "tiempo de florecimiento" (Guerra, 2017). Por lo tanto, el Pawkar raymi se ha vuelto una de las 4 celebridades más importantes en la cosmovisión andina del Ecuador, y esta celebridad se ha combinado con el Lalay Cañari (McGuffin, 2014; Matínez, 2015; Guerra, 2017).

Este ritual es netamente de origen Cañari, ya que no se ha observado alguna tradición similar en ninguna otra parte del Ecuador. Cabe destacar que estas denominaciones Pawkar raymi o Sisay pacha, no corresponden al idioma Cañari, por lo que, "El Lcdo. Jacinto Aguaiza menciona que son expresiones provenientes del kichwa, idioma Inca establecido en el actual Ecuador (J. Aguaiza, comunicación personal, febrero 4 de 2019). Además, el Kichwa se ha vuelto un idioma oficial del Ecuador (de Montecristi, A. C. 2008), y el resto de los idiomas como el Cañari ha ido perdiéndose (Ortiz, 2001). Por lo tanto, la verdadera denominación y la autenticidad del ritual también se ha perdido (Zaruma, 2006). Sin embargo, la población nativa Cañari aún utiliza palabras propias del idioma, además, se han mantenido topónimos, antropónimos, zoónimos y fitónimos propios (Quindi, 2018). Por lo tanto, es mediante estas palabras que se pretende descifrar los misterios del ritual Lalay Cañari, puesto que, en la actualidad, este está relacionada con mitos y leyendas (Ochoa, 2008). Asimismo, mediante la aplicación de encuestas y entrevistas en las comunidades nativas de Cañar, se obtuvo información para descifrar el contenido "oculto" de la tradición.

\section{Materiales y métodos}

El estudio se realizó en 4 áreas indígenas de Cañar (TUCAYTA, Sisid, Suscal y Socarte), localizadas en zona alta ( $2900 \mathrm{msnm}$ ) y zona baja (<2900 msnm). La provincia de Cañar limita al norte con la provincia Chimborazo, al sur con Azuay, al este con Azuay y Morona Santiago, y al oeste con Guayas (EcuRed, 2019).

Según el Censo Nacional (2010), la población en la provincia de Cañar fue de 225.184 habitantes (Foros, 2019). Mientras que la población autodefinida indígena fue de 6,7\% (Fernández, 2010), con alrededor de 15087 indígenas aproximadamente. Para aplicar encuestas y entrevistas, el tamaño de la muestra poblacional fue determinada basándose en la siguiente expresión:

\section{Fórmula 1. Muestra poblacional}

$$
\begin{aligned}
& \mathrm{n} \quad \mathrm{N} \times \mathrm{Z}_{\mathrm{a}}{ }^{2} \times \mathrm{p} \times \mathrm{q} \\
& =\mathrm{d}^{2} \times(\mathrm{N}-1)+\mathrm{Z}_{\mathrm{a}}{ }^{2} \times \mathrm{p} \times \mathrm{q} \\
& \text { Donde, } \\
& \\
& \\
& \mathrm{N}=\text { tamaño de la población. } \\
& \mathrm{Z}=\text { nivel de confianza. } \\
& \mathrm{p}=\text { probabilidad de éxito. } \\
& \mathrm{q}=\text { probabilidad de fracaso. } \\
& \mathrm{d}=\text { precisión. }
\end{aligned}
$$

\section{(Torres, et al., 2006, p.11)}

Basándose en esta fórmula, el tamaño de muestras fue considerada con 640 encuestas, con un nivel de confianza 95\% y el margen de error 5\%. Donde cada encuesta posee de 10 preguntas con 2 a 4 opciones de respuesta (Cuadro 1). Para la tabulación de datos se usó el programa informático Excel, y se realizó el análisis estadístico. 


\section{Cuadro 1. Encuesta para el ritual Lalay Cañari.}

\begin{tabular}{|c|c|}
\hline \multicolumn{2}{|c|}{ ENCUESTA LALAY CAÑARI } \\
\hline $\begin{array}{l}\text { Nombre: } \\
\text { Comunidad: }\end{array}$ & \\
\hline $\begin{array}{l}1^{\circ} \text { El carnaval Cañari es conocido como: } \\
\text { Pawkar raymi } \\
\text { Lalay Cañari } \\
\text { Otros }\end{array}$ & $\begin{array}{l}6^{\circ} \text { Los días del ritual son: } \\
3 \text { días } \\
1 \text { semana } \\
1 \text { mes }\end{array}$ \\
\hline $\begin{array}{l}2^{\circ} \text { Al paseador carnavalero Cañari se conoce como: } \\
\text { Taita carnaval } \\
\text { Paseador carnavalero } \\
\text { Otros }\end{array}$ & $\begin{array}{l}7^{\circ} \text { El canto de los paseadores se denomina: } \\
\text { Lalay } \\
\text { Wajay }\end{array}$ \\
\hline 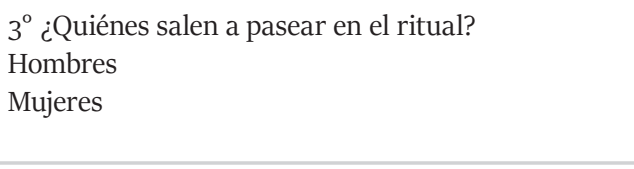 & $\begin{array}{l}8^{\circ} \text { Los jóvenes salen al ritual a: } \\
\text { Visitar sus amores } \\
\text { Realizar un paseo } \\
\text { Otros }\end{array}$ \\
\hline $\begin{array}{l}4^{\circ} \text { Los hombres, los paseadores carnavaleros son: } \\
\text { Niños } \\
\text { Jóvenes } \\
\text { Adultos } \\
\text { Ancianos }\end{array}$ & $\begin{array}{l}9^{\circ} \text { Durante el ritual ¿existe el Auka tuta? } \\
\text { SI } \\
\text { NO }\end{array}$ \\
\hline $\begin{array}{l}5^{\circ} \text { ¿Los padres autorizan a sus hijos para el ritual? } \\
\text { SI } \\
\text { NO }\end{array}$ & $\begin{array}{l}10^{\circ} \text { La indumentaria de paseadores representa: } \\
\text { Instrumentos de guerra } \\
\text { Simulacro al espíritu mítico }\end{array}$ \\
\hline
\end{tabular}

Fuente: Elaboración propia.

A modo de explicación, como se logra apreciar en la pregunta 1: Pawkar raymi es una de las 4 celebraciones andinas, proviene del Kichwa Pawkar "colorido", y raymi "fiesta", es decir "fiesta colorida". Por otro lado, Lalay Cañari, es un ritual milenario, proviene del Cañari Lalay "canto", es decir "el canto de los Cañari" (Cuadro 1). Mientras que en la pregunta 2: Taita carnaval está relacionado con un cerro mítico Cañari; proviene del Kichwa Taita "padre", es decir "padre carnaval" (Cuadro 1). Por otro lado, Paseador Carnavalero es un personaje disfrazado de Taita Carnaval, que pasea ambulando las comunidades (Figura 1). Ahora bien, en la pregunta 7: Lalay proviene del Cañari que significa "canto", y Wajay significa "entonar un instrumento de viento fabricado de hueso fémur" (Cuadro 1).

Y en la pregunta 9: Auka tuta se deriva del Kichwa Auka "guerrero o solado incásico", y tuta "noche", es decir "noche del soldado incásico o Cañari” (Cuadro 1).

\section{Resultados}

Los resultados se obtuvieron al extrapolar las encuestas y entrevistas, que fueron elaboradas de acuerdo al orden ceremonial del ritual. Empezando con la pregunta 1, el carnaval Cañari es conocido como: A) Pawkar raymi (50\%); B) Lalay Cañari (31\%); C) Otros (19\%), con diferencias significativas ( $\mathrm{P}:<0,05)$, resaltando que la opción A se diferencias estadísticamente de las demás (Tabla 1). Seguido de la pregunta 2, donde al paseador carnavalero se conoce como: A) Taita carnaval (51\%); B) Paseador carnavalero (25\%); C) Otros (24\%), con diferencias significativas (P:<0,05), de la misma manera, la opción A se diferencias estadísticamente de las demás (Tabla 1).

Por otro lado, en la pregunta 3, se indaga ¿Quiénes salen a pasear en el ritual? A) Hombres (84\%); B) Mujeres (16\%), con diferencias significativas (P:<0,05) (Tabla 1$)$. 
Seguidamente de la pregunta 4, de los hombres paseadores, los carnavaleros son: A) Niños (22\%); B) Jóvenes (70\%); C) Adultos (6\%); D) Ancianos (1\%), con diferencias significativas $(\mathrm{P}:<0,05)$, resaltando que la opción B se diferencia estadísticamente de las demás (Tabla 1). Adicionalmente, en la pregunta 5, se indaga ¿Los padres autorizan a sus hijos para el ritual? A) SI (91\%); B) NO (9\%), con diferencias significativas (P:<0,05) (Tabla 1).

En la pregunta 6, se indaga, los días del ritual son: A) 3 días (41\%); B) 1 semana (23\%); C) 1 mes (36\%), sin diferencias significativas (Tabla 1). Por su lado, en la pregunta 7, el canto de los paseadores se denomina: A) Lalay (90\%); y B) Wajay (10\%), con diferencias significativas ( $\mathrm{P}:<0,05)$ (Tabla 1). Además, en la pregunta 8, se indaga, los jóvenes salen al ritual a: A) Visitar sus amores (26\%); B) Realizar un paseo (44\%); C) Otros (30\%), con diferencias significativas (P:<0,05) (Tabla 1). Y en la pregunta 9, durante el ritual: ¿Existe el Auka tuta? A) SI (87\%); B) NO (13\%), con diferencias significativas $(\mathrm{P}:<0,05)$ (Tabla 1). Finalmente, en la pregunta 10, la indumentaria que llevan los paseadores representa: A) Instrumentos de guerra (73\%); B) Simulacro al espíritu mítico (27\%), con diferencias significativas (P:<0,05).

Tabla 1. Resultados por cada pregunta y significancia.

\begin{tabular}{|c|c|c|c|c|c|c|c|c|c|c|}
\hline Opciones & $\begin{array}{l}\text { Pregunta } 1 \\
(\%) \\
(\dot{\mathrm{X}} \pm \mathrm{DS})\end{array}$ & $\begin{array}{l}\text { Pregunta } \\
2(\%) \\
(\dot{\mathrm{X}} \pm \mathrm{DS})\end{array}$ & $\begin{array}{l}\text { Pregunta } \\
3(\%) \\
(\dot{\mathrm{X}} \pm \mathrm{DS})\end{array}$ & $\begin{array}{l}\text { Pregunta } \\
4(\%) \\
(\dot{\mathrm{X}} \pm \mathrm{DS})\end{array}$ & $\begin{array}{l}\text { Pregunta } \\
5(\%) \\
(\dot{\mathrm{X}} \pm \mathrm{DS})\end{array}$ & $\begin{array}{l}\text { Pregunta } 6 \\
(\%) \\
(\dot{\mathrm{X}} \pm \mathrm{DS})\end{array}$ & $\begin{array}{l}\text { Pregunta } 7 \\
(\%) \\
(\dot{X} \pm D S)\end{array}$ & $\begin{array}{l}\text { Pregunta } 8 \\
(\%) \\
(\dot{\mathrm{X}} \pm \mathrm{DS})\end{array}$ & $\begin{array}{l}\text { Pregunta } 9 \\
(\%) \\
(\dot{\mathrm{X}} \pm \mathrm{DS})\end{array}$ & $\begin{array}{l}\text { Pregunta } 10 \\
(\%) \\
(\dot{\mathrm{X}} \pm \mathrm{DS})\end{array}$ \\
\hline A & $50 \pm 5 b$ & $51 \pm 4 b$ & $84 \pm 3 b$ & $22 \pm 9 \mathrm{~b}$ & $91 \pm 4 \mathrm{~b}$ & $43 \pm 2$ & $90 \pm 9 \mathrm{~b}$ & $26 \pm 7 \mathrm{a}$ & $87 \pm 12 b$ & $73 \pm 2 b$ \\
\hline B & $31 \pm 18 \mathrm{ab}$ & $25 \pm 12 \mathrm{a}$ & $16 \pm 3 \mathrm{a}$ & $70 \pm 5 c$ & $9 \pm 4 \mathrm{a}$ & $21 \pm 18$ & $10 \pm 9 \mathrm{a}$ & $44 \pm 4 \mathrm{~b}$ & $13 \pm 12 \mathrm{a}$ & $27 \pm 2 \mathrm{a}$ \\
\hline C & $19 \pm 13 \mathrm{a}$ & $24 \pm 15 \mathrm{a}$ & & $6 \pm 6 \mathrm{a}$ & & $36 \pm 19$ & & $30 \pm 3 a$ & & \\
\hline D & & & & $1 \pm 1 \mathrm{a}$ & & & & & & \\
\hline EEM & 0,06 & 0,06 & 0,01 & 0,03 & 0,02 & 0,08 & 0,04 & 0,03 & 0,06 & 0,01 \\
\hline $\mathrm{P}$ & 0,0212 & 0,0135 & $<0,0001$ & $<0,0001$ & $<0,0001$ & 0,1632 & $<0,0001$ & 0,0022 & 0,0001 & $<0,0001$ \\
\hline
\end{tabular}

X: media estadística (promedio).

D.S: desviación estándar.

EEM: error estándar de la media.

P. $(<0,05)$ : existe diferencia estadística.

Fuente: Elaboración propia.

\section{Discusión}

No existe estudios previos y similares sobre el ritual Lalay Cañari. Por lo tanto, mediante la recopilación de información de habitantes nativos de este pueblo, se analizaron las preguntas de encuestas y entrevistas, donde se ha podido identificar palabras auténticas Cañari, que son usados a diario por los pobladores.

En la pregunta 1, el carnaval Cañari actualmente se conoce como Pawkar raymi (50\%), y se diferencia estadísticamente del resto de las opciones. Sin embargo,
Pawkar raymi proviene del Kichwa (procedencia Inca), y que es diferente al Cañari (J. Aguaiza, comunicación personal, febrero 4 de 2019). No obstante, como ya se mencionó, el ritual Lalay Cañari tiene su origen precisamente en el pueblo Cañari, (A. Quinde, comunicación personal, febrero 8 de 2019). Por otra parte, con la opción C (19\%), se reveló que en la zona baja de Cañar se conoce como "Sacha Chagrana", donde Sacha proviene del Cañari Zarza "mujer”, y del Kichwa Chagrana "cosechar". Por lo tanto, para los Cañari, el nombre de la festividad se sugiere "Zarza Chagray" que significa mujer que se dedica a cosechar durante una temporada. 
En cuanto al fin y al tiempo de esta labor, según la pregunta 6 con la opción C (36\%), los días del ritual antiguamente durarían un mes. Sin embargo, en la actualidad son 3 días (domingo, lunes y martes), y en la zona baja una semana. Es importante destacar la posible influencia de la religión con el miércoles de ceniza, forzando finalizar el ritual, siendo así, adoptando con el carnaval europeo (J. Pichasaca, comunicación personal, febrero 10 de 2019).

Ahora bien, ¿cuál fue el propósito del Zarza chagray? Mediante la pregunta 2 con la opción C (24\%), se ha podido revelar otro término "Muzu chungay" con lo que antiguamente denominarían a los paseadores carnavaleros, ya que los términos actuales son la combinación español y Kichwa. Por lo tanto, Muzu chungay o chuncay, proviene del Cañari Muzu "joven" y Chungay "juego ritual”, es decir, grupo de jóvenes que participan en un juego ritual. Por su lado, con la pregunta 8 y opción C (30\%), se ha podido entender cuál fue el propósito de esta actividad; los jóvenes Muzu chungas, salen con el propósito de realizar un entrenamiento militar, un procedimiento que en la antigüedad utilizarían con fines de preparar y adiestrar tácticas bélicas para librar batallas en servicio de la antigua nación Cañari. En la actualidad, según Manuel Pomaquiza (comunicación personal, febrero 14 de 2019), el ritual es celebrado por jóvenes que salen a pasear o visitar familiares (44\%), y/o visitar o buscar sus amores (26\%).

Además, con las preguntas 3, 4 y 10; se argumenta, que las personas que salen al ritual son hombres (84\%); y de estos (70\%) son jóvenes. Además, la indumentaria usada es considerada instrumentos de guerra por un $73 \%$; mientras el otro $27 \%$, lo relaciona con la indumentaria de un espíritu mítico; Apu de un cerro. En la actualidad, el Lalay Cañari es una celebración realizada por organizaciones indígenas con el fin de rescatar y conservar antiguas tradiciones (A. Quinde, comunicación personal, febrero 8 de 2019). Donde participan con los siguientes porcentajes según las respuestas: mujeres (16\%), niños (22\%), adultos (6\%) y ancianos (1\%). Sin embargo, jóvenes de las comunidades milenarias Quilloac, Cuchucun, Shizho, Sisid, Suscalpamba, Milmil, Kullauko y Socarte, aún realizan el ritual día y noche midiendo su fuerza y resistencia. Por lo tanto, desde la antigüedad hasta la actualidad, los padres son los que autorizan y dan bendición a sus hijos para el ritual (91\%).

Desde hace varias décadas, por la migración familiar, muchos jóvenes no cuentan con sus padres quienes otorgan el permiso (9\%), por lo que los jóvenes salen al ritual utilizando su propia intuición (J. Pichasaca, comunicación personal, febrero 10 de 2019). En este sentido, en los jóvenes Cañari nace la intuición, el entusiasmo y la dedicación innata de prepararse por sus propios méritos, ya que conseguir o fabricar la indumentaria y los instrumentos idóneos requiere de un gran sacrificio (A. Quinde, comunicación personal, febrero 8 de 2019). Entonces, mediante el ritual, los jóvenes desarrollarán sus destrezas y habilidades. Estos demuestran ante la sociedad la madurez, donde entran al servicio de su nación y, además, los jóvenes que superen la travesía del ritual, también demuestran a los taitas (mayores) que se encuentran en forma enérgica y aptos para desenvolverse en el campo rústico, así, aprovechan esta cualidad para enamorar a sus pretendientes y, a su vez, lograr la aceptación de los padres.

Por su lado, las mujeres con el Zarza chagray, contribuyen realizando preparativos y festines alimenticios destinados a los Muzu chungas, donde los principales beneficiados del gran festín son los jóvenes del ritual y, así, terminan enamorando o adquiriendo su pareja (L. Aguaiza, comunicación personal, febrero 17 de 2019). Sin embargo, los Muzu chungas, para beneficiarse del gran festín y recibimiento por las Zarza chagras en sus hogares, deben interpretar los 12 cantos de Lalay (90\%), y adicionalmente, saber entonar el Wajay (10\%) (J. Duy, comunicación personal, febrero 22 de 2019). Lalay provienen del Cañari que significa "canto" y Wajay "entonar un instrumento de viento fabricado de hueso fémur”. En este contexto, según Isidoro Pichasaca (comunicación personal, febrero 29 de 2019), narra el Lalay de entrada para visitar una casa hogar (Lalaytapish yachashpa, wajaytapish yachashpa, sukta killa lamarta, sukta killa urkuta shamumuni "sabiendo el canto, sabiendo entonar instrumentos de viento, he venido $y$ he llegado seis meses por tierra y seis meses por mar"), y al escuchar este canto, las Zarzas tienen la costumbre de preguntar los 12 cantos, cada canto tiene relación con un mes específico, vinculado a su vez 
con personas, naturaleza, un ser mítico, o una deidad, y así, indican cada acontecimiento del ritual (Ochoa, 2008). Por consiguiente, el canto del lalay Toro (antiguamente taruga "venado") es un cántico de enfrentamiento entre grupos combatientes (I. Pichasaca, comunicación personal, febrero 29 de 2019). Por otro lado, es necesario responder por qué estos cantos se relacionan con lo mítico. Según Jacinto Aguaiza (comunicación personal, febrero 4 de 2019), en la cosmovisión andina no existe el cielo ni el infierno; para los Cañari, el espíritu después de la muerte se transforma, por lo mismo, para los Yachak (sabios), existe en dimensiones divididas del cosmos, donde se encuentra conexo con todo aquello que lo rodea en un mismo espacio-tiempo, siendo así como se explica que, en los ojos de algunos habitantes, estos seres míticos aparecen.

En este contexto, el ritual desde su creación, nunca fue un acto pagano, idólatra ni de diversión, más bien, fue un acto de sacrificio hacia la nación y una forma de rendir culto a la Diosa Luna, debido a que la travesía antiguamente duraba un mes, precisamente en la última luna del año, ya que los Cañari manejaban el calendario lunar, y finalizaba con el Auka tuta (87\%), donde, al día siguiente, empezaría el nuevo año andino conocido como Mushuk nina. Es así como, durante esta temporada del ritual, las Zarzas se dedicaban profundamente a recibir a los Muzus en sus hogares y alimentarlos con frutas, cuy y chicha (bebida), donde los Muzu chungas participan del gran festín, y el sobrante lo guardan en su piksha (bolso) para continuar la travesía día y noche por todos los rincones, probando su fuerza y midiéndose a sí mismos, debido a que el camino guardaría grandes presagios y peligros. A pesar de esto, los Muzu chungas están preparados militarmente y armamentísticamente (K. Cungachi, comunicación personal, marzo 2 de 2019). Los Muzu chungas llevan como indumentaria de protección la Sombredera o lalay muchiku (especie de escudo cilíndrico fabricado de cuero de venado o de cóndor), suspendida con hilos (posiblemente Qhipus, numeración Inca), de un peso promedio de $5 \mathrm{~kg}$, el cual, es insertada en la cabeza y que protege este miembro (Figura 1). Además, llevan el Zamarhu (especie de pantalón fabricado de cuero con fibra natural de llama o alpaca), de un peso promedio de $6 \mathrm{~kg}$, que protege las extremidades inferiores. Por otro lado, como armas de guerra llevan la
Waraka (concha de mar) suspendida del antebrazo por un hilo tejido, y el chicote. Asimismo, como instrumentos de música portan el pingullo (flautín), fabricado de carrizo, que sirve para entonar diferentes melodías agudas, la entonación Gallo (antiguamente cóndor) por ejemplo, señala el inicio de enfrentamiento entre combatientes. Adicionalmente, llevan el Wajuro (hueso fémur fabricado como flauta grave), de un peso promedio de $6 \mathrm{~kg}$, el cual sirve como instrumento de música y también de guerra. Por último, llevan una Barza o wankar "tambor" (conocido también como caja). Este fabricado con aros de madera, donde su interior está hecho de un lado sonoro de cuero de llama y del otro un lado lírico, hecho a base de cuero de puma con vibradoras, con los hilos de sujeción para aros fabricado con este mismo cuero enrollado, o también con hilo tejido de fibra de llama o alpaca. El instrumento posee un promedio de $3 \mathrm{~kg}$ (A. Quinde, comunicación personal, febrero 4 de 2019) (Figura 1). De este modo, los Muzu chungas tocan la Barza con entonaciones de batalla, marcando el paso o ritmo militar característico y armónico, donde, la Barza percibe el espíritu de la madre tierra que alienta al poseedor. En este sentido, toda la indumentaria de un Muzu chunga pesaría alrededor de $25 \mathrm{~kg}$ sobre cada individuo, por lo mismo, fabricar estos instrumentos requiere de gran responsabilidad (A. Quinde, comunicación personal, febrero 4 de 2019). En la actualidad, la fabricación de los instrumentos y la indumentaria resulta más fácil, ya que se utiliza otro tipo de materiales.

En cuanto al juego ritual, los Muzu chungas militarmente, perfeccionan el Pukara "combatientes", donde el primer grupo que logre realizarlo es el vencedor, es decir, el Pukara es rodear y encerrar en círculo al grupo opositor y comprimir de afuera hacia adentro hasta derribar, utilizando todos los instrumentos de guerra que llevan sobre ellos, donde el rito termina, muchas veces, con derramamiento de sangre y muerte, más aún en la última noche de la travesía llamada el Auka tuta, conocido como la noche de muerte. Es así como en la muerte provocada durante esta última noche no existe justicia (L. Aguaiza, comunicación personal, febrero 17 de 2019). Antiguamente, el derramamiento de sangre y muerte fue una forma de rendir culto a la diosa Luna, donde los afortunados morían librando batallas con honor y gloria. 
Cabe destacar que para las madres Cañari, la pérdida de sus descendientes resultaría irreparable, por lo que muchas madres abniegan enviar a sus hijos al ritual (F. Solano, comunicación personal, febrero 17 de 2019). No así los padres, que por sus principios del deber y del servicio hacia la nación, afirmar que el deber es cumplir con el ritual, y de esta manera, asegurar la estirpe Cañari con bravura y coraje, y así, los enemigos de la nación llegarían a conocer la gran preparación militar, manteniéndose al margen conservando sus límites.

Según el padre Ángel María Iglesias (1985), dentro de la nación existían cacicazgos que constantemente libraban batallas entre sí. Por lo que se sugiere la puesta en práctica de estos juegos rituales. No obstante, al acechar el peligro en común, estas fuerzas se unían practicando la dualidad (zona baja y zona alta), y luego aglomerando distintas regiones de la nación hasta conformar un ejército numeroso e irrevocable. Siendo así que, en cada ciudadano Cañari, el ritual implica una gran responsabilidad. Como sus ancestros, los Cañari forjarían feroces guerreros, inspirados en los míticos Apus que susurraban voces desde las piedras milenarias, que bajaban desde los altos cerros para alentar a ser hombres libres, hacia una victoria, a no rendirse jamás, a proteger su tierra y su familia, y a morir por el servicio de su nación, esa era su promesa, su juramento y la ley Cañari. Se logra afirmar, entonces, que el principal propósito del ritual Muzu chungay fue formar guerreros profesionales y nobles de corazón, que posteriormente los llamarían los legendarios "Bravos Cañaris” y, también, a través de mitos y leyendas llegarían a conocer como Taita carnaval, una fuerza militar que resultaría difícil o imposible de conquistar.

Según la historia de González Suárez (1878), en su libro "Estudio Histórico Sobre los Cañaris", se sabe que a los Incas, en su campaña de conquista hacia las tierras ecuatorianas, les resultó difícil conquistar a los Cañari. Por lo tanto, en su primer intento de invasión, los Cañari derrotaron al Inca Tupac Yupanqui y su ejército en Guapondelig, (actual Cuenca). Posteriormente, el Inca regresa a Cusco y años más tarde, reúne un ejército jamás visto de 150 mil soldados aproximadamente, y apuñala el corazón de Guapondelig. Sin embargo, el general Cañari "Cacique Duma" retrocede hacia Hatun Cañar (actual
Cañar) y resiste con todos sus súbditos. Finalmente, el Inca Tupac sin querer derramar más sangre opta por otras estrategias, donde, decide unir naciones realizando lazos matrimoniales entre el Inca y la princesa de Duma, donde, según historiadores ecuatorianos nacería Inca Huayna Capac (padre de Atahualpa). Posteriormente, para la invasión española también resultaría difícil derrotar a los Cañari, ya que las enfermedades del viejo mundo, fueron las responsables del exterminio de la población nativa de Cañar, con las que lograron disminuir fuerzas, y así someter e invadir tierras Cañari (Albornoz, 1946; Uberem, 1974; Idrovo, 1985; Iglesias, 1985; Murillo y Santos, 2017).

\section{Conclusiones}

Mediante antiguas usanzas, con palabras propias Cañari, que se encuentran dispersas en todo este territorio, se ha podido entender el ritual Lalay Cañari, y se sugiere la denominación "Muzu chungay", un ritual o usanza destinado para los jóvenes de este pueblo con la finalidad de un entrenamiento militar, que celebrarían en la festividad conocida como "Zarza chagray".

Para seguir corroborado con los avances de los antiguos Cañari, podrían proponerse nuevos estudios enfocados en los cánticos (lalays), en las entonaciones con el pingullo Cañari, en los acontecimientos ocurridos durante el Zarza Chagray, durante el ritual Muzu Chungay, e involucrar todos los pobladores Cañari-Kichwa hablantes de todo el territorio de la antigua nación.

\section{Agradecimientos}

A TUCAYTA y a todas las comunidades nativas, que mantienen vivas las costumbres y tradiciones Cañari. A todas las personas entrevistadas, donde se encuentra oculto todos los conocimientos ancestrales. 


\section{Bibliografía}

Albornoz, V. (1946). La antigua Tomebamba y Cuenca que nace (Recuperación de libro y patrimonio). Universidad de Cuenca, Ecuador, MCMXLVI, 7 $-158$.

Alulema, L., y Guamán, W. (2012). Incidencia de la mujer Cañari de la parroquia Socarte (General Morales) en la pervivencia cultural y en el desarrollo comunitario, durante el período 2005-2012 (Tesis de Licenciatura). Universidad Estatal de Bolivar, Cuenca - Ecuador, 178.

Alulema, R., y López, M. (2017). Cosmovisión andina Cañari y determinacion social de la salud. Investigación Talentos, 2, 80.

Arizala, M., y Merchán, J. (2010). Proyecto de investigación y producción de un documental fotográfico sobre la arquitectura, áreas poblacionales, sitios arqueológicos y rutas de acceso de la Cultura Cañari (Tesis de Licenciatura). Universidad Politécnica Salesiana. Cuenca, 182. Obtenido de https://dspace.ups.edu.ec/ handle/123456789/1253

Burgos, H. (2003). La identidad del pueblo Cañari: Deconstrucción de una nación étnica. Abya Yala(1), 5-90.

Butler, I. M. (1997). Zapoteco de Yatzachi (No. 37). Mexíco: Instituto Lingüístico de Verano.

CIDAP. (2016). Las fajas tejen la historia cañari. El tiempo(62), 1-3.

Córdova, C. (2013). Fajas tradicionales del austro ecuatoriano (Tesis licenciatura). Universidad de Azuay. Cuenca, 182. Obtenido de http://dspace. uazuay.edu.ec/handle/datos/2591

de Montecristi, A. C. (2008). Constitución de la República del Ecuador. Obtenido de http://biblioteca. defensoria.gob.ec/handle/3700o/823
EcuRed. (2019). Provincia de Cañar (Ecuador). Obtenido de https://www.ecured.cu/Provincia_de_ Ca\%C3\%Biar_(Ecuador)

Encalada, O. (2017). El cantado cuencano es cañari. $E l$ Tiempo. Diario de Cuenca.

Fernández, W. (2010). Las Cifras de las Nacionalidades y Pueblos Indígenas: mirada desde el Censo de Población y Vivienda 2010. Obtenido de Academia: http://www.academia.edu/11296235/_ Las_Cifras_de_las_Nacionalidades_y_Pueblos_ Ind $\% \mathrm{C}_{3} \%$ ADgenas_mirada_desde_el_Censo_ de_Poblaci\%C3\%B3n_y_Vivienda_2010

Foros. (2019). Habitantes de Ecuador por Provincias. Obtenido de Ecuador.ec: http:// www.forosecuador.ec/forum/ecuador/ educaci\% $\% \mathrm{C}_{3} \%$ B3n-y-ciencia/9004-habitantesde-ecuador-por-provincias

Gómez, S. (2003). Atlas de historia de América. Caracas: LIMUSA.

González Suárez, F. (1878). Estudio histórico sobre los Cañaris, antiguos habitantes de la provincia del Azuay. Quito: CLERO.

Guerra, J. (2017). Fotoreportaje que evidencia la riqueza cultural andina del Pawkar Raymi en Peguche Imbabura (Tesis de Licenciatura). Universidad Politécnica Salesiana, Ecuador, 30. Obtenido de http://dspace.ups.edu.ec/ handle/123456789/1456o

Gutierrez, A., y Siguencia, P. (2017). Diseño de productos gráficos basados en la iconografía Cañari como medio de difusión cultural (Tesis de Licenciatura). Escuela Superior Politécnica de Chimborazo. Riobamba, 170.

Hollaway, A. (2015). Ingapirca: Proof that the Inca Respected the Cultures of those they Conquered. Obtenido de ANCIENT ORIGINS: https://www. ancient-origins.net/ancient-places-americas/ ingapirca-proof-inca-respected-cultures-thosethey-conquered-004175 
Idrovo, J. (1985). Primera fase de conquista incásica en los Andes septentrionales. Los Cañaris y la conquista incásica del Austro Ecuatoriano. En Tomebamba (pág. 71). Quito: Abya Yala.

Iglesias, A. M. (1985). Los Cañaris: aspectos históricos y culturales (1985 ed.). Azogues: Consejo Provincial de Cañar.

Lynch, T., y Pollock, S. (1989). Chobshi Cave in Retrospect. Andean Past, 2(4), 33.

Magaña, E. (2006). Astronomía de algunas poblaciones Quechua-Aymara del Loa Superior, Norte de Chile. Boletín del Museo Chileno de Arte Precolombino, 11(2), 65.

Marín, A. (2017). Diseño interior a partir de los rasgos de la iconografía y cosmovisión Cañari: fase Cashaloma (Tesis de Licenciatura). Universidad del Azuay. Cuenca, 95. Obtenido de http://dspace. uazuay.edu.ec/handle/datos/7096

Matínez, A. (2015). Fiesta en latitud cero. Revista Latinoamericana de Investigación en Organizaciones, Ambiente y Sociedad , 6(7), 3.

McGuffin, L. (2014). Rituales de reciprocidad en los carnavales indígenas andinos (Tesis Máster). Louisiana State University and Agricultural and Mechanical College, 125. Obtenido de https:// digitalcommons.lsu.edu/gradschool_theses

Miller, P. (2013). MARAS ¿Observatorio Astronómico Cañari? Obtenido de Revista Cuenca Ilustre - Ecuador: https://patomiller.wordpress. com/2013/o $/ 28 /$ maras-observatorioastronomico-canari/

Morocho, M., y Zaruma, W. (2012). Recuperación y análisis de la música del taita carnaval en el Cantón Cañar (Tesis Licenciatura). Universidad de Cuenca, Ecuador, 52. Obtenido de http://dspace.ucuenca. edu.ec/handle/123456789/356
Murillo, P., y Santos, L. (2017). La invasión inca al actual Ecuador y la resistencia indígena de los pueblos del norte a finales del siglo XV (Tesis Licenciatura). Universidad central del Ecuador, 27-103. Obtenido de http://www.dspace.uce.edu. ec/handle/2500o/11383

Naula, P. (2018). Las fajas: símbolo de energía en la cultura cañari. Obtenido de Diario El Tiempo: http://documentacion.cidap.gob. ec:808o/bitstream/cidap/1791/1/Las\%20 fajas \% 2os\% $\% 3 \%$ ADmbolo\% $\%$ ode $\% 20$ energ $\% \mathrm{C}_{3} \% \mathrm{ADa} \% 2$ oen $\% 2$ ola $\%$ 2ocultura $\% 20$ ca\% $\% \mathrm{C}_{3} \%$ B1ari.pdf

Ochoa, M. (2008). Clasificación y análisis cultural de los mitos cantados en la fiesta mítica del Pawkar Raymi Cañari (Tesis Máster)). Universidad de Cuenca, Ecuador. Obtenido de http://dspace. ucuenca.edu.ec/handle/123456789/16911

Ortiz, G. (2001). El quichua en el Ecuador: ensayo históricolingüístico (2001 ed.). Riobamba: Abya-Yala.

Quindi, A. (2018). Lengua Cañari. Riksinakuy, 1. Obtenido de https://atuplan.com/?s=Andr\%C3\%Ags+Quindi

Torres, M., Paz, K., y Salazar, F. (2006). Tamaño de una muestra para una investigación de mercado. Boletín electrónico, 2, 1-13.

Uberem, U. (1974). Los Cañaris y la conquista española de la sierra ecuatoriana otro capítulo de las relaciones interénicas en el siglo XVI. Journal de la Société des Américanistes, 63, 263-274.

Zaruma, V. (2006). Wakanmay, aliento sagrado: perspectivas de teología india: una propuesta desde la cultura cañari (2006 ed.). Quito: AbyaYala.

Zhungri, R. (2017). Lalay Raymi, una investigación desde la vivencia Rosa Zhungri. Repositorio Digital CIDAP, 5 . 


\section{Anexos}

Figura 1. Taita carnaval y/o paseador carnavalero Cañari.

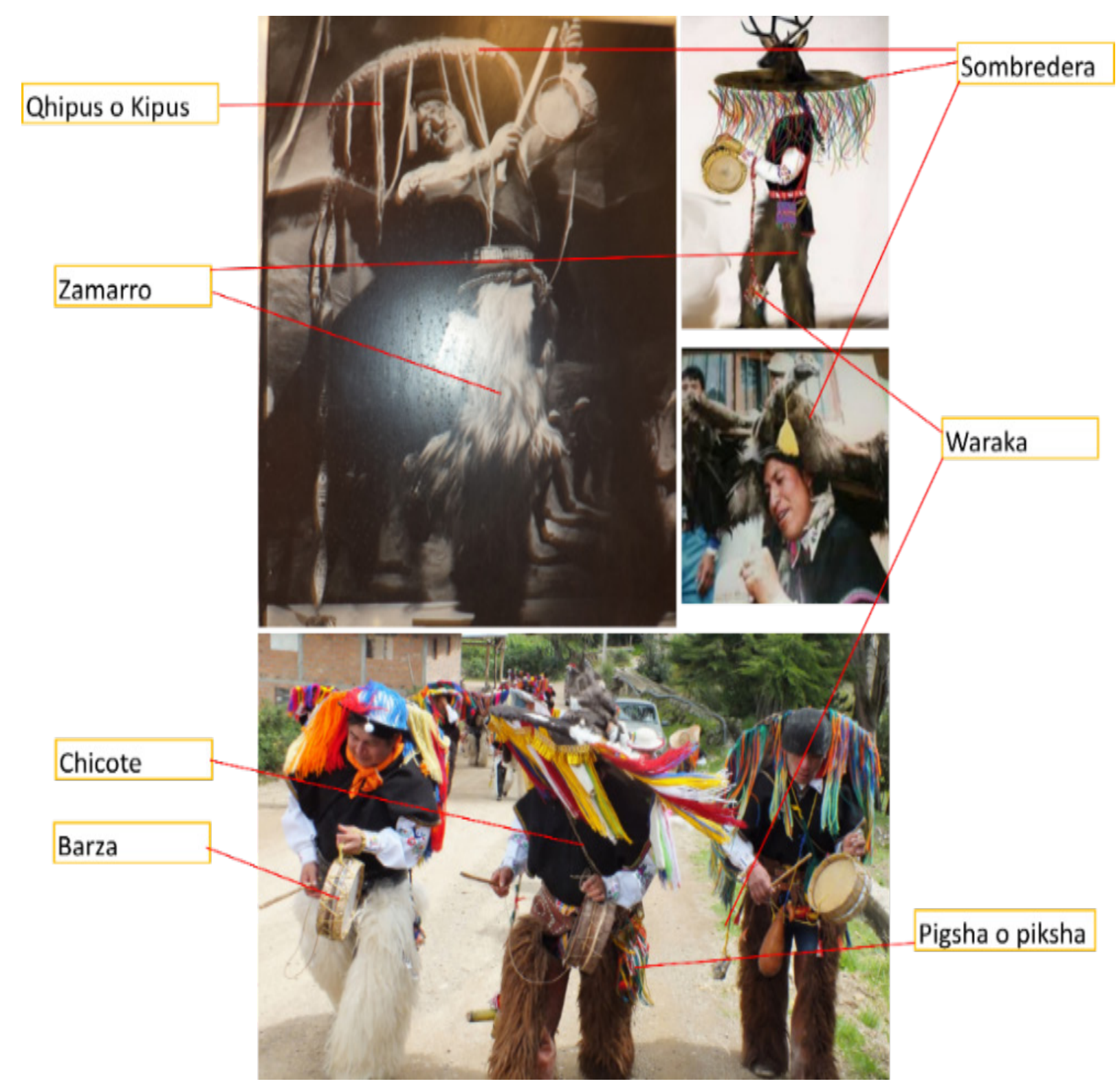

Fuente: Elaboración propia, basado en la imágenes de taita carnaval con personajes como Pedro Solano y Enrique Pomavilla. 